\title{
Thick Galactic Cosmic Radiation Shielding Using Atmospheric Data
}

\author{
Robert C. Youngquist ${ }^{\mathrm{a}, *}$, Mark A. Nurge ${ }^{\mathrm{a}}$, Stanley O. Starr ${ }^{\mathrm{a}}$, and Steven L. Koontz ${ }^{\mathrm{b}}$ \\ ${ }^{a}$ Mail-Stop NE-L5, National Aeronautics and Space Administration (NASA), Kennedy Space Center, FL 32899 \\ ${ }^{\mathrm{b}}$ Mail-Stop ES-411, National Aeronautics and Space Administration (NASA), Johnson Space Center, TX 77058 \\ * Corresponding author. Tel. 1-321-867-1829 (USA), Fax 1-321-867-1177 (USA). E-mail: Robert.C.Youngquist@nasa.gov
}

NASA is concerned with protecting astronauts from the effects of galactic cosmic radiation and has expended substantial effort in the development of computer models to predict the shielding obtained from various materials. However, these models were only developed for shields up to about $120 \mathrm{~g} / \mathrm{cm}^{2}$ in thickness and have predicted that shields of this thickness are insufficient to provide adequate protection for extended deep space flights. Consequently, effort is underway to extend the range of these models to thicker shields and experimental data is required to help confirm the resulting code. In this paper empirically obtained effective dose measurements from aircraft flights in the atmosphere are used to obtain the radiation shielding function of the earth's atmosphere, a very thick shield. Obtaining this result required solving an inverse problem and the method for solving it is presented. The results are shown to be in agreement with current code in the ranges where they overlap. These results are then checked and used to predict the radiation dosage under thick shields such as planetary regolith and the atmosphere of Venus.

\section{Keywords - Galactic Cosmic Radiation, Radiation Protection, Radiation Shielding, Inverse Problem, Atmospheric Radiation}

\section{INTRODUCTION}

Even before Apollo, the dangers of deep-space radiation were recognized, and significant effort has since been spent in quantifying this radiation, in understanding its effects on humans, and in finding ways to ameliorate it - and we now know that the situation is more perilous than previously thought. There are still unknowns, but the recent report (2012) from the National Academies on NASA Space Technology Roadmaps and Priorities summarizes the situation: “...models predict that crewed missions beyond low Earth orbit (LEO) would be limited to three months or less because of adverse health impacts...." [1] Yet three months is insufficient for manned space flight beyond LEO, except for lunar sorties, causing deep-space missions to be presently unachievable. Radiation effects pose such a fundamental obstacle to man's exploration of the solar system that the National Academies state that "Space Radiation Health Effects" (including investigating protection technologies) should be NASA's second highest technical challenge (behind "Improving Access to Space").

The majority of this health detriment is caused by the accumulated effect of galactic cosmic radiation (GCR). GCR is composed of high-energy nuclei ranging from protons to uranium that enter the solar system from all directions. When these "projectiles" encounter matter, either in the structure of a spacecraft or within a human body, they collide with and shatter nuclei, causing a shower of secondaries composed of charged particles, neutrons, and gamma rays - all of which are damaging to human tissue. Neurons are harmed irreparably, cataracts can start to develop, and the risk of dying from cancer, i.e. the risk of exposureinduced death (REID) rises [2].

With this picture in mind, researchers in the 1990s began to extol the benefits of hydrogen-containing materials as shields against GCR, because hydrogen has no neutrons to add to the secondaries and it is the most effective material per unit mass at slowing down ions [3]. For a few years it appeared that these materials might provide adequate shielding, and projects were funded to study high-hydrogen-containing materials, especially polyethylene [4]. But the models of the early 2000 s did not adequately account for the interaction of radiation with the human body and its tissues. Refinements in these models led to predictions of high levels of radiation $[2,5,6]$ and are the basis for the statement quoted by the National Academy in the introductory paragraph above. It was shown that adding substantial polyethylene could actually increase the effective radiation dose received by an astronaut and that $120 \mathrm{~g} / \mathrm{cm}^{2}$ of polyethylene (more than a meter of material) could not provide the shielding necessary to allow a mission to Mars [see Fig. 6.5 in ref 2].

In the face of this picture a reasonable question to ask would be, "How much shielding is sufficient to protect an astronaut for a given mission?" However, even though the various radiation codes are highly developed [7] they have only been verified against in-flight and accelerator data out to about $120 \mathrm{gm} / \mathrm{cm}^{2}$ and beyond this the models use exponential extrapolations. Model verification data to high shielding mass is needed to have confidence for spacecraft and surface habitat design. One of us 
(Koontz) recognized that such data might be obtained by manipulating information from the Federal Aviation Authority (FAA). The FAA has developed a CARI-6 computer program that supplies GCR effective dose values for any location in the world from ground level up to 60000 feet $[8,9]$ and is available as a free download. The data within the CARI- 6 is a combination of transport code, human body modeling, and dosimeter measurements [10] and represents real world measurements of the effective dose an individual receives from galactic cosmic radiation when protected by a thick atmospheric shield. The problem is then to extract shielding functions for the earth's atmosphere from the CARI-6 data, requiring solving an interesting inverse problem.

\section{THEORETICAL ANALYSIS}

The data supplied by the CARI-6 program describes the effective radiation dose received by a human from galactic cosmic radiation, which is isotropic, arriving at the earth equally from all directions. Yet, to an observer at some height $h$ above ground level, this total effective radiation, $R(h)$, is the sum of radiation that is not spatially uniform, having passed through different mass lengths of the earth's atmosphere. Mathematically this can be expressed as

$$
R(h)=\int_{0}^{2 \pi} \int_{0}^{\pi / 2} S(m(\theta, \phi, h)) \sin (\theta) d \theta d \phi
$$

where $S(m)$ is the effective radiation dosage per steradian received by the observer after having passed through an atmospheric mass, $m(\theta, \phi, h)$, (units of mass/area) where $\theta$ is the elevation angle, measured from the zenith as shown in Figure 1 , and $\phi$ is the azimuthal angle. In the discussion below $4 \pi S(m)$ will be referred to as the atmospheric radiation shielding function since this corresponds to the total effective radiation dose seen when surrounded by a spherically symmetric shell of atmosphere.

The elevation angle in Equation 1 is only integrated to $\pi / 2$ radians, corresponding to the horizon and accounting for the radiation shielding provided by the planet itself. Also, in all cases of interest in this paper the atmospheric mass will be assumed to be independent of azimuth, allowing equation (1) to be rewritten as

$$
R(h)=2 \pi \int_{0}^{\pi / 2} S(m(\theta, h)) \sin (\theta) d \theta
$$

Mathematically, the problem here is to solve for the function $S(m)$ assuming $R(h)$ is known. Problems such as this are referred to as inverse problems and commonly arise in 3-D imaging problems, such as tomography [11] and sonar [12], but can appear in diverse fields. A common approach to solving these problems, which we will develop below, is to find a way to rewrite the integral as a matrix multiplication and then calculate the inverse matrix.

The atmospheric mass function, $m(\theta, h)$, represents the amount of atmospheric mass between an observer located at a height $h$ above the ground, and a point above the atmosphere when looking at an angle $\theta$ down from the zenith; along the $s$ direction shown in Figure 1. In order to obtain an expression for this function we will assume an isothermal atmosphere in which pressure and density decay exponentially with increasing height. The analytic expressions in this model are given by

$$
P(h)=P(0) \exp [-h / H] \quad \text { and } \quad \rho(h)=-P^{\prime}(h) / g=(P(0) /(g H)) \exp [-h / H]=\rho(0) \exp [-h / H]
$$

where $P(h)$ is the pressure as a function of height, $\rho(h)$ is the atmospheric density as a function of height, $g$ is the gravitational acceleration $\left(9.8 \mathrm{~m} / \mathrm{sec}^{2}\right)$, and $H$ is the scale height. The scale height of the atmosphere is given by $H=k T /(M g)$ where $k$ is Boltzmann's constant $\left(1.38 \times 10^{-23} \mathrm{~J} / \mathrm{K}\right), T$ is the average atmospheric temperature in Kelvin (we use $260 \mathrm{~K}$ as an average atmospheric temperature), and $M$ is the mean molecular mass of dry air (equal to the effective number of atomic mass units in the atmosphere (29) times $1.66 \times 10^{-27} \mathrm{~kg}$ ). These values yield a scale height of 7600 meters. In addition we will assume that the pressure at ground level, $P(0)$ is given by 101,000 Pascals. 


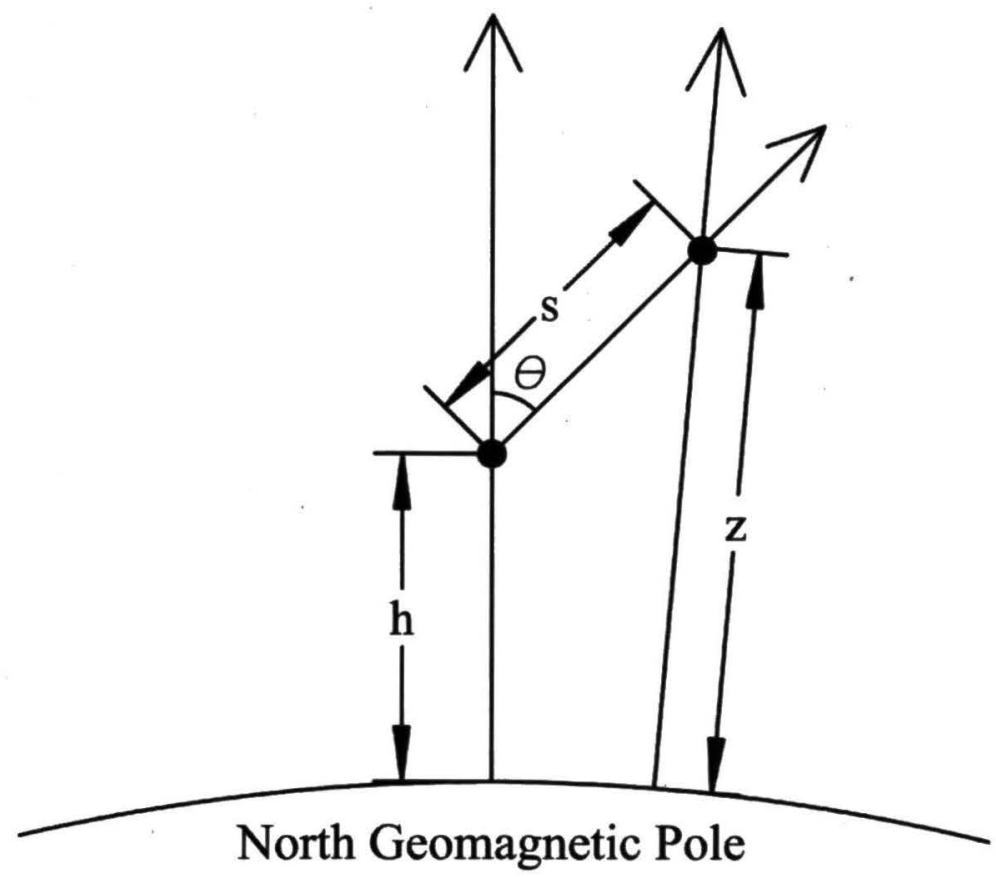

Figure 1. This figure defines the variables used in the calculation of the atmospheric mass function.

Now, referring to Figure 1, the mass function is given by the integral of the atmospheric density along the line of sight direction shown by the $s$ parameter. Writing this explicitly and incorporating the density function in Equation (3) yields

$$
m(\theta, h)=\int_{0}^{\infty} \rho(s) d s=\int_{0}^{\infty} \rho(0) \exp [-z(\theta, s) / H] d s .
$$

However, the density is a function of height, $z$, and the integral is over the line of sight variable, $s$, so we need to determine the function $z(\theta, s)$. This can be done by using the law of cosines and noting that all upward directed vectors pass through the center of the earth,

$$
z(s, \theta)=-r_{e}+\sqrt{h^{2}+2 h r_{e}+r_{e}^{2}+s^{2}+2\left(h+r_{e}\right) s \cos (\theta)} .
$$

where $r_{c}$ is the radius of the earth, $6.378 \times 10^{6}$ meters. Combining equations (4) and (5) yields the final expression for the atmospheric mass function

$$
m(\theta, h)=\rho(0) \int_{0}^{\infty} \exp \left[\left(r_{e}-\sqrt{h^{2}+2 h r_{e}+r_{e}^{2}+s^{2}+2\left(h+r_{e}\right) s \cos (\theta)}\right) / H\right] d s
$$

Figure 2 shows three plots of the mass function, for heights of 0,9000 meters, and 18000 meters above the ground versus angle from vertical. As the angle approaches the horizon at $\pi / 2$ radians the mass increases rapidly as expected. Note that this plot is in units of $\mathrm{g} / \mathrm{cm}^{2}$, rather than the $\mathrm{SI} \mathrm{kg} / \mathrm{m}^{2}$, because most of the radiation shielding literature uses CGS rather than MKS units. 


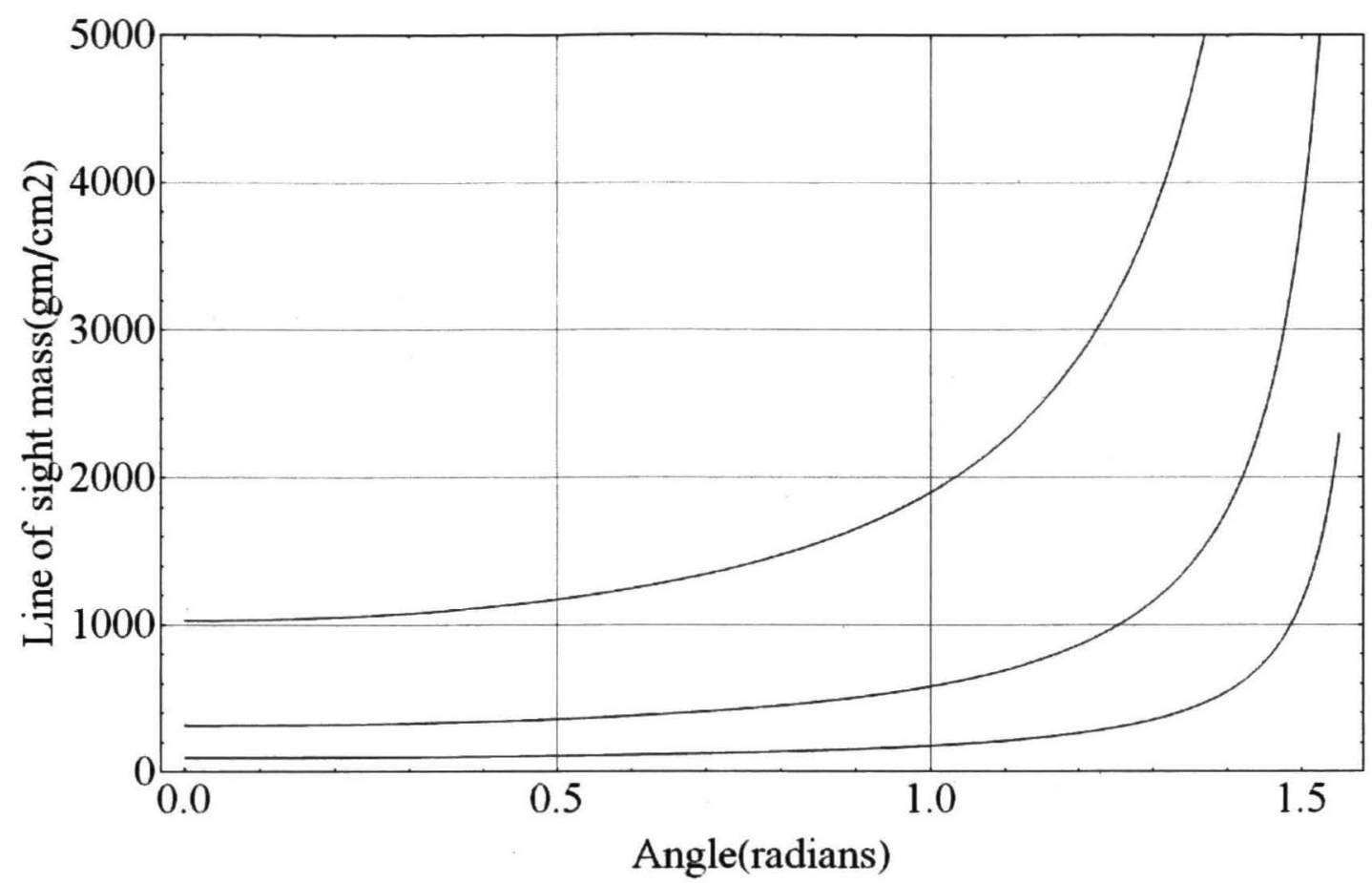

Figure 2. Plots of the total atmospheric mass between an observer and space versus the angle in radians measured down from vertical. The highest plot corresponds to ground level, the middle plot is at a height of $9000 \mathrm{~m}$, and the lowest plot is at a height of $18000 \mathrm{~m}$.

Having derived an expression for the atmospheric mass function, the inverse problem given in Equation (2) can now be converted into a matrix expression. Start by selecting a set of discrete masses, $m_{i}$, based on the masses provided by Equation (6), such that the radiation shielding function, $S(m)$, can be approximated as being constant over each mass interval. Let $m_{1}$ be the smallest mass and $m_{n+1}$ be the largest so that there are $n$ intervals and the radiation shielding function, $S(m)$ can be approximated as $S\left(m_{i}\right)$ for each interval between masses $m_{i}$ and $m_{i+1}$. Now chose a discrete height, $h_{j}$, and for each discrete atmospheric mass, $m_{i}$, at that height let $\theta_{i}$ be the angle that corresponds to that mass. So between each angle interval, $\theta_{i}$ to $\theta_{i+1}$, the atmospheric mass, $m_{i}$, seen by an observer looking at that angle is approximately constant. In addition, if at some height the masses are too small to occur then set the corresponding $\theta_{i}=0$ and if the masses are too large then set the corresponding $\theta_{i}=\pi / 2$. Doing this allows Equation (2) to be rewritten as

$$
R\left(h_{j}\right)=2 \pi \sum_{i=1}^{n} S\left(m_{i}\right) \int_{\theta_{i}}^{\theta_{i+1}} \sin (\theta) d \theta=2 \pi \sum_{i=1}^{n} S\left(m_{i}\right)\left(\cos \left(\theta_{j, i+1}\right)-\cos \left(\theta_{j, i}\right)\right)=\sum_{i=1}^{n} S\left(m_{i}\right) \Theta_{j, i}
$$

where the matrix $\Theta_{j, i}$ describes the angular ranges at each height, $h_{j}$, through which an observer sees radiation corresponding to $S\left(m_{i}\right)$. Writing this in matrix notation yields

$$
\vec{R}=\Theta \vec{S}
$$

which can be solved to obtain the atmospheric radiation shielding function, $4 \pi S(\mathrm{~m})$, but care is required. Simply inverting the matrix, $\Theta_{j, i}$, in Equation (8) can yield chaotic results because small noise or errors in the input data, $R\left(h_{j}\right)$, can produce large variations in the output data, $S\left(m_{i}\right)$. The present case is no exception and its explicit solution will be discussed in the next section. 


\section{CARI-6 DATA ANALYSIS}

The FAA's CARI-6 computer program supplies the effective dose values for any location in the world, from ground level up to 60000 feet, averaged over any month going back to 1958 . For the purposes of the present study we chose our location to be the north geomagnetic pole ( 80 degrees $\mathrm{N}, 72$ degrees $\mathrm{W}$ ) in order to minimize the impact of the earth's magnetic field on the propagation of the GCR. We then chose two months, June of 2000, corresponding to a solar maximum where the sun's magnetic field was strong, reducing the amount of GCR that reaches the earth, and June of 2009, corresponding to a solar minimum where the GCR impact on the earth was high. We tabulated this data for every 2500 feet from 2500 feet to 50000 feet (we dropped the ground point because it appeared to have a background radiation contribution) and then every 1000 feet from 50000 feet up to 60000 feet. The interval was decreased at the higher elevations to help reduce uncertainty in the lower mass range. This data is shown in Figure 3 with the lower plot corresponding to solar maximum and the upper plot corresponding to solar minimum.

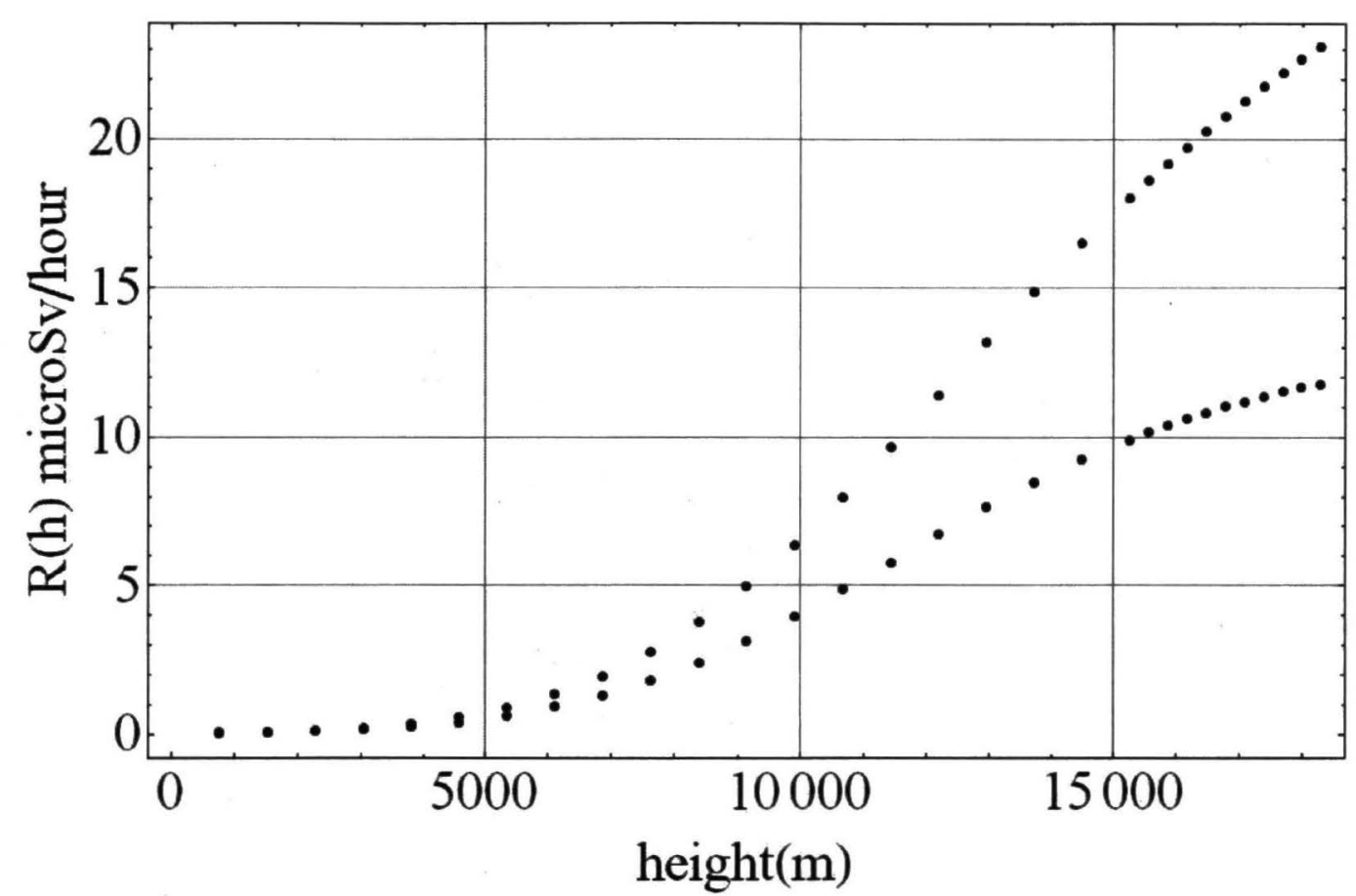

Figure 3. This is the CARI-6 effective radiation doses from GCR versus height at the north geomagnetic pole for June 2000 , a solar maximum when the radiation levels were low, and for June 2009, a solar minimum when the radiation levels were high.

A polynomial fit to the CARI-6 data was found in order to supply effective radiation doses at arbitrary heights. Then, using the results of the theory section, a discrete matrix form for the inverse problem was developed allowing the inverse problem to be solved using the Mathematica routine "LeastSquares". This specifies that the square root of the sum of the squares of the differences between the fit and the data be used as a norm to find the best solution to the inverse matrix problem. Doing this yielded a pair of atmospheric radiation shielding functions, one for solar maximum and one for solar minimum. These two functions are the primary results of this effort and are shown in Figures 4 and 5.

Figures 4 and 5 show the effective radiation dose that an astronaut would receive in deep space when surrounded by a shell of atmosphere with a given mass density. By deep space we mean a location well outside of the earth's protective atmosphere and magnetic field, yet at a distance from the sun similar to that of the Earth. This form is typical of that found in the literature and allows a direct comparison with previously published results. For example Cucinotta [13-Fig. 6.6] shows the effective dose for a male behind an aluminum shield with masses ranging from 0 to $120 \mathrm{~g} / \mathrm{cm}^{2}$. For solar maximum his results show an effective radiation dose between $270 \mathrm{mSv} /$ year and $320 \mathrm{mSv} /$ year (depending on the model used) at $120 \mathrm{~g} / \mathrm{cm}^{2}$ shielding. Our solar maximum plot is consistent with this result showing an effective radiation dosage of $276 \mathrm{mSv} /$ year at $120 \mathrm{gm} / \mathrm{cm}^{2}$. However, our results extend beyond $120 \mathrm{~g} / \mathrm{cm}^{2}$ showing a peak radiation dose in the $160-180 \mathrm{~g} / \mathrm{cm}^{2}$ range of $287 \mathrm{mSv} /$ year. For solar 
minimum Cucinotta shows a radiation dosage of between $430 \mathrm{mSv} /$ year and $520 \mathrm{mSv} /$ year at $120 \mathrm{~g} / \mathrm{cm}^{2}$ mass. Our results are larger, see Figure 5, showing about $580 \mathrm{mSv} /$ year at $120 \mathrm{~g} / \mathrm{cm}^{2}$. This reason for this discrepancy is unclear, but might be due to differing choices of solar minimum.

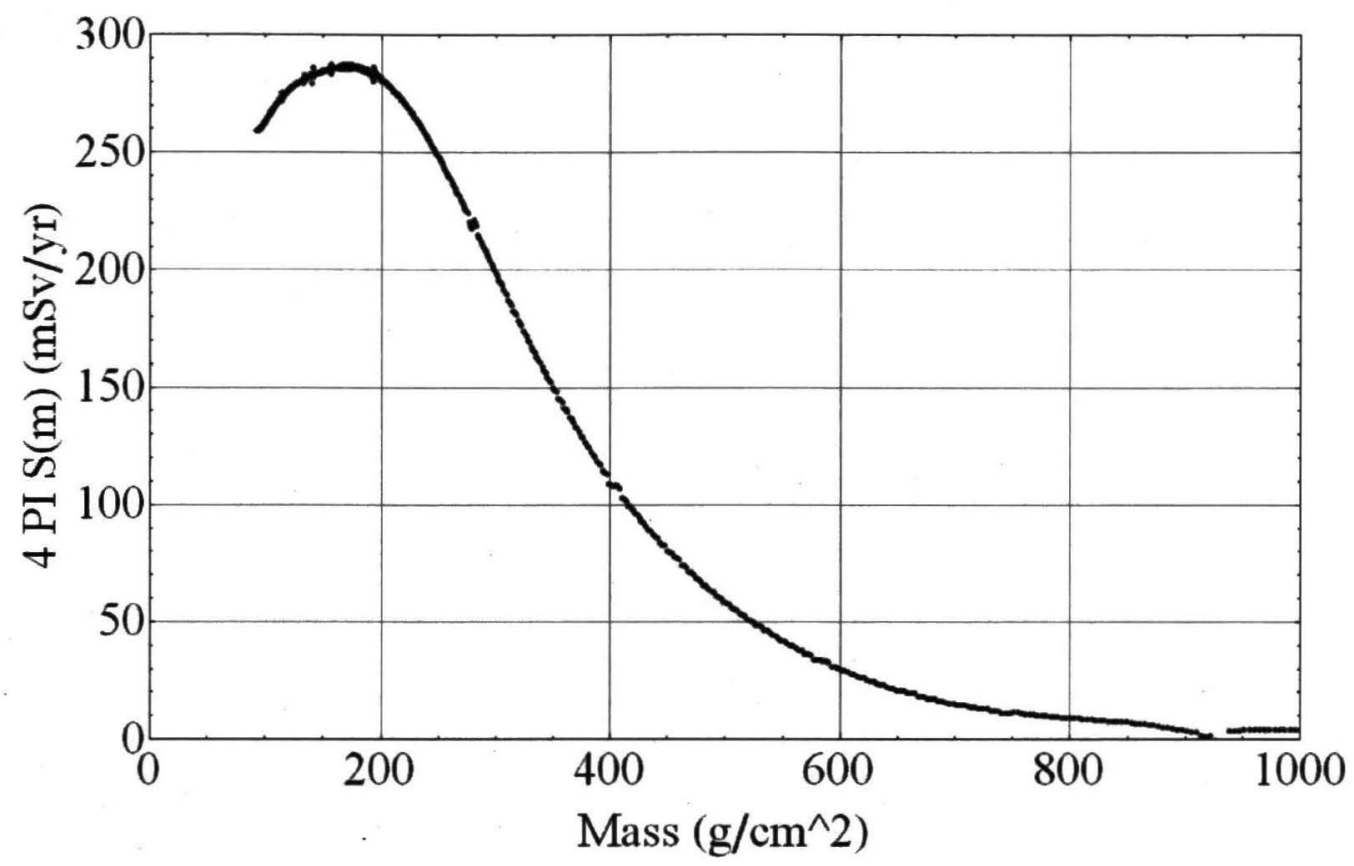

Figure 4. This plot shows the atmospheric radiation shielding function at solar maximum, i.e. this is the radiation dose an astronaut would receive in deep space at solar maximum when surrounded by a shell of atmosphere of given mass thickness.

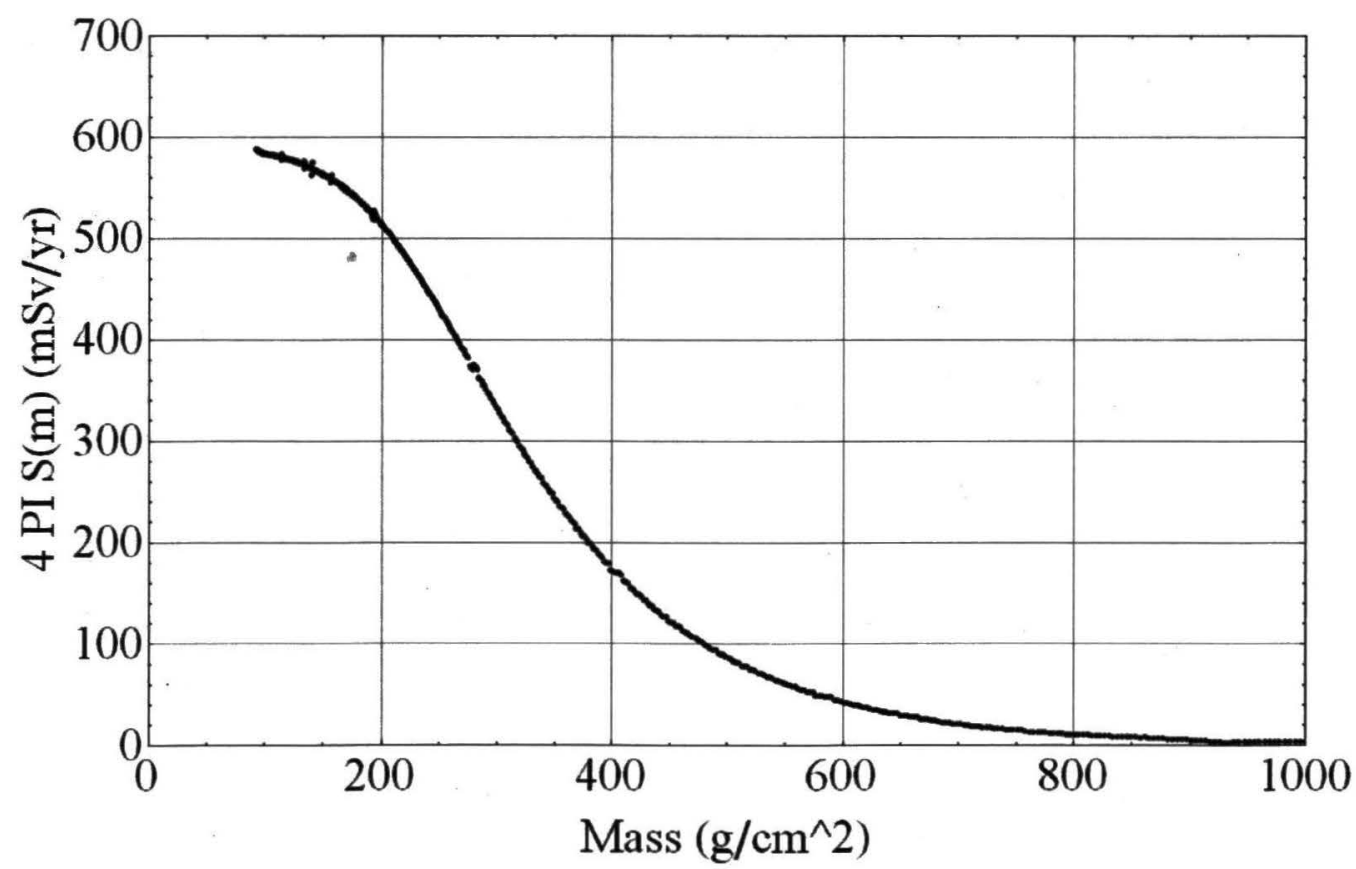

Figure 5. This plot shows the atmospheric radiation shielding function at solar minimum, i.e. this is the radiation dose an astronaut would receive in deep space at solar minimum when surrounded by a shell of atmosphere of given mass thickness. 
The unique feature of the atmospheric radiation shielding functions shown in Figures 4 and 5 is that they provide data on the high mass shielding of the atmosphere. For atmospheric masses larger than $200 \mathrm{~g} / \mathrm{cm}^{2}$ the curves are roughly exponential, decaying about a factor of 3 for every $150 \mathrm{~g} / \mathrm{cm}^{2}$ of additional mass. Also, these results are applicable to shielding materials other than the constituents of the earth's atmosphere. Studies have shown that most non-hydrogen containing materials have similar shielding properties [see, Figure 4 in reference 5] when compared by mass density. Consequently, the atmospheric radiation shielding functions in Figures 4 and 5 can be used with a reasonable degree of confidence to predict the shielding effectiveness of other materials. For example, a question commonly raised by the space community is, "How much regolith is needed on the Moon to protect astronauts for long time periods?" These plots show that $1000 \mathrm{~g} / \mathrm{cm}^{2}$ is more than sufficient to provide long term radiation protection. So, if one intended to use the lunar regolith as a radiation shield and its density is $3 \mathrm{~g} / \mathrm{cm}^{3}$ then a layer between 3 and 4 meters thick would provide maximal protection with no need to dig deeper.

NASA is currently considering setting $150 \mathrm{mSv}$ as a maximum allowable lifetime dose for an astronaut and these curves indicate the amount of shielding that would be necessary to achieve that for a given time period in deep space. The resulting amounts of material may be excessive for use as shielding on a spacecraft, though a recent publication has considered this possibility [14].

\section{Checking the Result and Predicting the Radiation Dosage in the Venusian atmosphere}

Before considering other planetary atmospheres, we begin with Earth in order to check our results and to test the forward calculation process. Inserting the atmospheric radiation shielding functions into Equation 2, the radiation dosage seen at various heights over the north geomagnetic pole can be calculated. The results are shown in Figure 6 along with the CARI-6 data, demonstrating that the shielding plots found through the inverse method described above do indeed yield the correct effective radiation dosages when used in the forward problem.

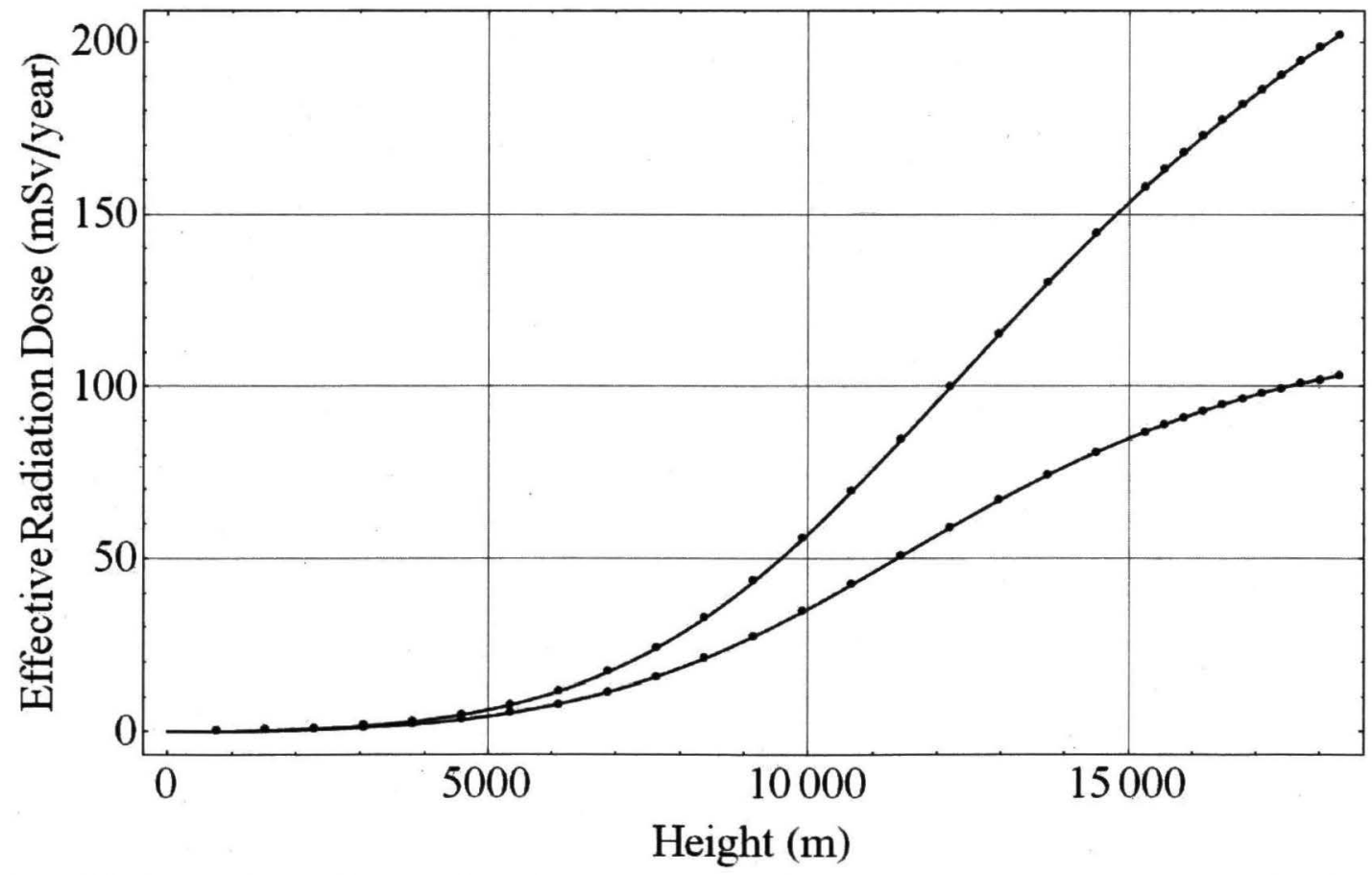

Figure 6. This plot shows the effective radiation dosage for heights above the geomagnetic north pole on Earth, for solar minimum (the upper plot) and for solar maximum (the lower plot). The points are the CARI-6 data. This plot shows that the solutions to the inverse problem can be used in the forward direction to recover the original data. 
Now that the forward process has been verified for Earth's atmosphere we can proceed to model the total effective radiation dosage that an astronaut would receive at various heights in the atmosphere of Venus. We chose Venus to study because other entities within the solar system either have no appreciable atmosphere (e.g. Mercury, Mars) or have primarily hydrogen atmospheres (e.g. Jupiter) for which the above analysis is not applicable or have an atmosphere but are at a much larger distance from the sun where the GCR radiation levels are not known (e.g. Titan). For clarification, Mars has a thin atmosphere that might provide some shielding. We calculated the effective radiation dosage for an explorer on the surface using Cucinotta's results for angles where the atmospheric mass is less than $100 \mathrm{gm} / \mathrm{cm}^{2}$ and our results for angles (near the horizon) where the total atmospheric mass is thick. Our analysis showed that the radiation dosage on the surface of Mars is roughly half of that in deep space, due to the planet itself blocking half of the sky and the atmosphere providing essentially no shielding (we did not calculate the additional radiation caused by GCR interaction with the ground). So for the purposes of radiation shielding the Martian atmosphere is ineffective.

Table 1 shows the pertinent data needed to perform atmospheric effective radiation dosage calculations for Venus where Earth and Mars are included for comparison. The average atmospheric temperature, the mean molecular mass, and the gravitational acceleration are needed in order to calculate the scale height of the respective atmosphere. Using this with the surface pressure allows the surface density to be calculated and then using the body radius in Equation (6) allows the shielding mass to be found. Equation 2 can then be used to determine the total effective radiation dosage versus height within that atmosphere.

\begin{tabular}{|l|l|l|l|}
\hline & Earth & Mars & Venus \\
\hline Average Atmos. Temp K & 260 & 210 & $300 *$ \\
\hline Mean Molecular Mass (AMU) & 29 & 43.3 & 43.4 \\
\hline Gravitational acceleration $\left(\mathrm{m} / \mathrm{s}^{2}\right)$ & 9.81 & 3.7 & 8.87 \\
\hline Scale height $(\mathrm{m})$ & 7600 & 10900 & $6500^{*}$ \\
\hline Radius $(\mathrm{m})$ & 6378000 & 3396000 & 6051000 \\
\hline Surface pressure $(\mathrm{Pa})$ & 101000 & 636 & $107700 *$ \\
\hline Surface density $\left(\mathrm{kg} / \mathrm{m}^{\wedge} 3\right)$ & 1.35 & 0.016 & $1.87 *$ \\
\hline Notes/issues & & $\begin{array}{l}\text { Lowest point is }-7 \\
\text { km. }\end{array}$ & $\begin{array}{l}* 50 \mathrm{~km} \text { above the } \\
\text { actual surface }\end{array}$ \\
\hline
\end{tabular}

Table 1. The parameters required to derive the Galactic Cosmic Radiation shielding characteristics of various atmospheres.

Venus is sometimes called Earth's sister planet and this relationship applies to the two atmospheres, assuming the comparison with Earth is done 50-55 kilometers above the sủrface of Venus. The surface of Venus is over $700 \mathrm{~K}$ and is extremely hostile, but at a height of about 50-55 kilometers the Venusian atmosphere has similar pressure and temperature to the Earth's atmosphere at ground level. The benign condition at this location has caused at least one researcher [15] to suggest colonizing Venus, referring to it as the most Earthlike environment in the solar system. So it is not surprising that the effective radiation dose starting $50 \mathrm{~km}$ above the surface of Venus point is very similar to Earth's - compare Venus's effective radiation dose curve shown in Figure 8 to Earth's shown in Figure 6. A floating manned science platform on Venus could range between 50 and 57 $\mathrm{km}$ above the surface for years without exceeding the lifetime radiation dose suggested by NASA. 


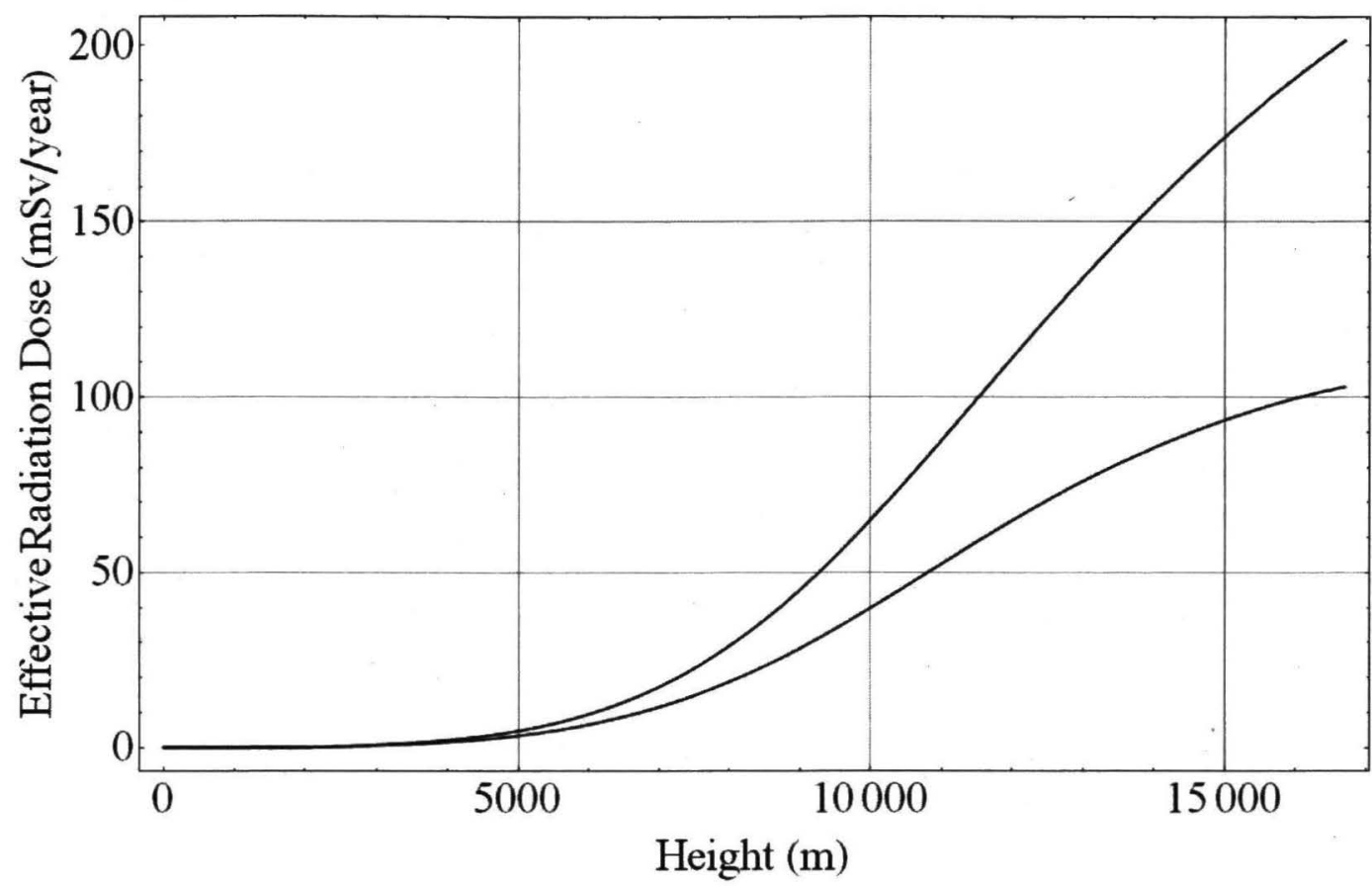

Figure 8. These are plots of the effective radiation dose within the atmosphere of Venus versus height (starting at 50 kilometers above the surface) for solar maximum (lower plot) and solar minimum (upper plot).

\section{CONCLUSION}

We have shown that by using effective radiation dosage data from the FAA's CARI-6 program that the atmospheric shielding of the Earth's atmosphere can found. To our knowledge this is the first time that an effective radiation shielding curve has been found for very thick, up to $1000 \mathrm{~g} / \mathrm{cm}^{2}$, mass densities. This information can provide verification for computer models used to predict material shielding properties. In addition, it supplies useful insight into how much material on the moon or Mars would be required to construct an effective radiation shield and how much shielding would be required to protect an astronaut in deep space. It also allows modeling of the effective radiation dosages expected within the atmosphere of Mars and Venus. As might be expected, the Martian atmosphere provides very little radiation protection; however, there is a benign region in Venus' atmosphere, ranging between $50 \mathrm{~km}$ and $57 \mathrm{~km}$ above the ground where there is a high degree of radiation protection.

\section{REFERENCES}

1. National Research Council of the National Academies. NASA Space Technology Roadmaps and Priorities, Restoring NASA's Technical Edge and Paving the Way for a New Era in Space. The National Academies Press. Washington, D.C. 2012.

2. Cucinotta, F. A., Kim, M.-H. Y., and Chappel, L. J. ,"Space Radiation Cancer Risk Projections and Uncertainties-2010," NASA/TP-2011-216155, National Technical Information Service, July 2011.

3. Wilson, J.W., Cucinotta, F.A., Kim, M.-H.Y., and Schimerling, W., "Optimized Shielding for Space Radiation Protection," International Workshop on Space radiation Research and $11^{\text {th }}$ Annual NASA Space Radiation Health Investigator's Workshop, Arona (Italy) May 27-31, 2000, Physica Medica-Vol. XVII, Suplement 1, 2001.

4. Guetersloh, S., et. al. "Polyethylene as a radiation shielding standard in simulated cosmic environments," Nuclear Instruments and methods in Physics research Section B: Beam Interactions with Materials and Atoms, vol. 252, Issue 2, November 2006, pp. 319-332.

5. Cucinotta, F. A., Kim, M.-H. Y., and Ren, L., "Evaluating shielding effectiveness for reducing space radiation cancer risks," Radiation Measurements, Vol. 41, (2006) pp. 1173-1185.

6. Durante, M. and Cucinotta, F. A., "Physical basis of radiation protection in space travel," Reviews of Modern Physics, Vol. 83, Oct-Dec. 2011. Pp. 1245-1281.

7. Heinbockel, J. H., et. al. "Comparisons of Radiation Transport Codes, HZETRN, HETC, and FLUKA, Using the 1956 Webber SPE Spectrum,” NASA/TP-2009-215560, National Technical Information Service, January 2009. 
8. Friedberg, W. et. al. "Radiation Exposure During Air Travel: Guidance Provided by the Federal Aviation Administration for Air Carrier Crews, "Health Physics, Vol. 79, Issue 5, Nov. 2000, pp. 591-595.

9. McCall, M. J., et. al. “Development of a Predictive Code for Aircrew Radiation Exposure," Radiation Protection Dosimetry, vol. 136, No. 4, July 17, 2009, pp. 274-281.

10. Hajek, M., et. al. "Dose Assessment of Aircrew using Passive Detectors," Radiation Protection Dosimetry, vol. 100, No. 14, July 1, 2002, pp. 511-514.

11. Olafsson, G., Quinto, E. T., editors, The Radon Transform, Inverse Problems, and Tomography. Proceedings of Symposia in Applied Mathematics, vol. 63. American Mathematical Society. Providence, Rhode Island, 2006.

12. Taroudakis, M. I., and Makrakis, G. N., editors, Inverse Problems in Underwater Acoustics. Springer-Verlag, New York. 2001.

13. Cucinotta, F. A., Kim, M.-H. Y., and Chappel, L. J. ,"Space Radiation Cancer Risk Projections and Uncertainties-2012," NASA/TP-2013-217375, National Technical Information Service, January 2013.

14. Koontz, S. L., et. al. "Estimating the Effects of Astronaut Career Ionizing Radiation Dose Limits on Manned Interplanetary Flight Programs," $43^{\text {rd }}$ International Conference on Environmental Systems, AIAA, July 14-18, 2013.

15. Landis, G. A., "Colonization of Venus," Space Technology and Applications Int. Forum-STAIF 2003, Feb. 2-5 2003, AIP Conference Proceedings, Vol. 654, pp. 1193-1198. 

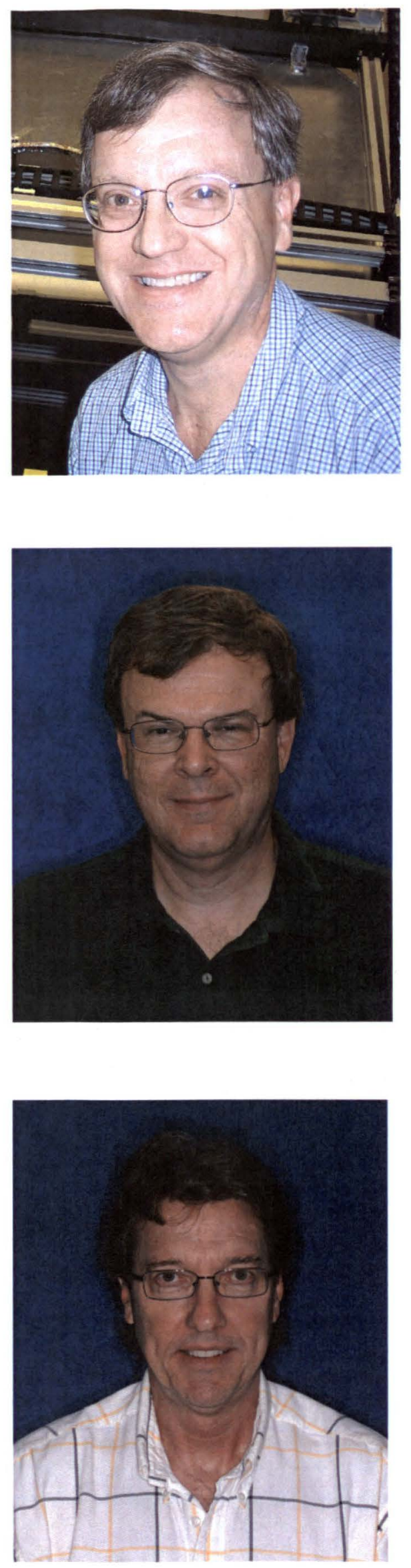

Robert C. Youngquist received M.S. and $\mathrm{Ph}$.D. degrees from Stanford University in Stanford, California in 1982 and 1984, respectively, in the field of applied physics.

He was a faculty member in the department of Electronic and Electrical Engineering at University College London from 1984 to 1986. After this he joined the Kennedy Space Center (as a contractor until 1999 and then as a NASA employee) where he has spent more than 20 years developing ground support tools for the Space Shuttle in addition to working on a diverse range of topics in his role as lead of the KSC Applied Physics Lab.

Mark Nurge is a physicist and electrical engineer at NASA's John F. Kennedy Space Center (KSC) Applied Physics Laboratory. He has worked at KSC since 1987 as an engineer, manager, and now a scientist, specializing in sensors and instrumentation.

Prior to joining NASA, he earned Bachelor and Master of Science degrees in electrical engineering from the Georgia Institute of Technology in 1985 \& 1986, respectively. Since then, he has earned M.S. degrees in engineering management (1992) and physics (2003) and a Ph.D. in physics (2007) from the University of Central Florida.

Stanley O. Starr received an M.A. degree in Physics from the University of South Florida, Tampa, in 1978.

He has worked at the Kennedy Space Center for 31 years in various capacities including engineering and physics. His duties have primarily involved developing technologies in support of the Space Shuttle Program. He is currently Branch Chief for the KSC Applied Physics Lab, a group involved with developing advanced space flight technologies. 


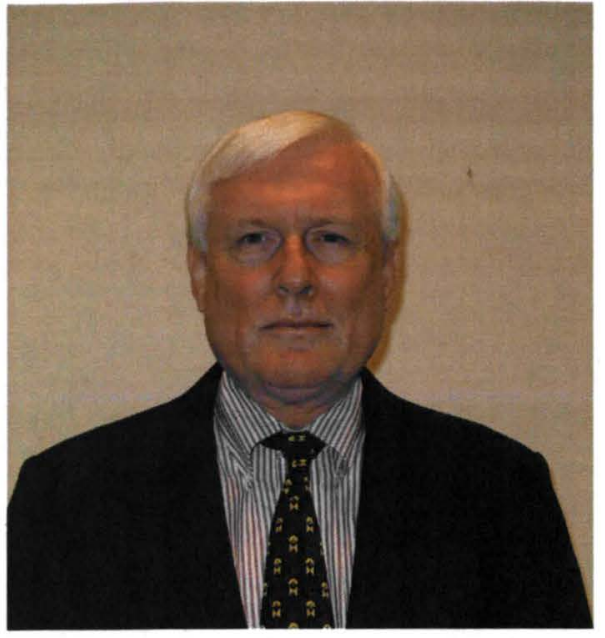

Steve Koontz received a B.S. degree in chemistry from the University of California at Berkeley and a Ph.D. degree in chemistry from the University of Arizona at Tucson in 1983.

Dr. Koontz has worked for 24 years at the Johnson Space Center and is the International Space Station Manager for Space Environments. His primary responsibility is the safety, reliability, and mission success of the ISS with regard to radiation and contamination. This work has provided data allowing the verification of space radiation transport and modeling codes and has led to several publications. 